\begin{tabular}{ll|l}
\cline { 2 - 3 } & \multicolumn{2}{l}{ Intervent Neurol 2013;2:193-200 } \\
\cline { 2 - 3 } & $\begin{array}{l}\text { DOI: 10.1159/000363070 } \\
\text { Publisned onIIne: June 27, 2014 }\end{array}$ & $\begin{array}{l}\text { (C) 2014 S. Karger AG, Basel } \\
1664-9737 / 14 / 0024-0193 \$ 39.50 / 0 \\
\text { www.karger.com/ine }\end{array}$ \\
\hline
\end{tabular}

\title{
Cerebrospinal Venous Outflow in Multiple Sclerosis Patients versus Fatigue and/or Depression
}

\author{
Marloes H.J. Hagens Erwin L.J. Hoogervorst Stephan T.F.M. Frequin \\ Selma C. Tromp \\ Department of Neurology, St. Antonius Hospital, Nieuwegein, The Netherlands
}

\section{Key Words}

Chronic cerebrospinal venous insufficiency - Multiple sclerosis - Colour Doppler sonography · Quality of life · Fatigue · Depression

\begin{abstract}
Background: Endovascular treatment of impaired cerebrospinal venous outflow has been suggested to improve the overall quality of life in multiple sclerosis (MS) patients. Fatigue and depression are key factors in measuring the quality of life in MS patients. Objective: In the present study, we investigated the correlation between anomalous venous outflow and the seriousness of fatigue and depression in MS patients and healthy controls. Methods: Five cerebrospinal venous outflow parameters were measured in 20 MS patients and age- and sexmatched controls using extra- and transcranial Colour Doppler sonography. All patients and volunteers filled out the Fatigue Severity Scale (FSS) and Hospital Anxiety Depression Subscale (HADS). Results: Nine abnormal parameters were found in 8 MS patients, whereas five abnormal parameters were found in 3 healthy controls (no significant difference). Only $1 \mathrm{MS}$ patient met the criteria for chronic cerebrospinal venous insufficiency compared to 2 healthy controls. No significant differences were found in the FSS and HADS scores between patients with and without abnormal cerebrospinal venous outflow parameters. Conclusions: We found no significantly impaired cerebrospinal venous outflow in patients with MS versus sex- and age-matched controls. Furthermore, we did not find any correlation between anxiety or depression and impaired venous outflow in MS patients.




\section{Introduction}

The pathogenesis of multiple sclerosis (MS) is still not fully understood. It is widely accepted that an immune-mediated process causes inflammation and demyelination of the central nervous system in MS. Much research is being done to get a better understanding of what triggers and sustains this inflammatory process. The often perivascular, especially perivenous, location of the white matter lesions in MS might indicate a vascular involvement in its pathophysiology. This led the Italian vascular surgeon Zamboni and his colleagues [1] to a new theory linking abnormal cerebrospinal venous outflow to the pathogenesis of MS. They suggested that chronic cerebrospinal venous insufficiency (CCSVI) is strongly associated with relapsing-remitting MS (RRMS) and primary progressive MS (PPMS). The diagnosis of CCSVI is based on extra- and transcranial Colour Doppler sonographic examination and venography. CCSVI can be caused by various venous anomalies (stenosis or obstructions) resulting in an altered extracranial venous outflow, and therefore, changed cerebral venous haemodynamics [2]. How this correlates with the pathogenesis of MS has been discussed ever since this theory was first published in 2009.

In the following years, many researchers have tried to confirm these spectacular results with strikingly variable outcomes. A meta-analysis by Laupacis et al. [3] showed a likelihood of finding CCSVI in MS patients compared to controls ranging widely from more than a 200-fold increased risk to even a decreased risk.

Even though the relevance of anomalous venous outflow in the pathogenesis of MS remains unclear, Zamboni et al.'s [1] spectacular results inspired many different MS centres to perform endovascular treatments. The clinical effect of these treatments on patients' neurological status is commonly measured by the Expanded Disability Status Scale (EDSS) and the annualised relapse rate. These outcomes vary widely in different studies, making an interpretation difficult. Overall, according to the results of several studies available today, a significant effect of endovascular treatment of CCSVI on the objective neurological status has not been proven. On the other hand, a significant improvement of patients' (subjective) quality of life has been suggested in several studies [4-6]. The effect on the quality of life has been measured using various different instruments like the Multiple Sclerosis Quality of Life-54 instrument (MSQoL54), the Multiple Sclerosis Impact Scale-29 (MSIS-29) and non-standardised questionnaires. Of these different outcomes, fatigue has been described to be one of the most common and invalidating factors in MS patients. As much as $65-97 \%$ of MS patients suffer from fatigue, limiting them in multiple aspects of everyday life [7-10]. Fatigue is also associated with several psychosocial problems in MS patients. Malagoni et al. [11] found a dramatic reduction of perceived chronic fatigue in MS patients with CCSVI after reestablishment of cerebral venous return suggesting that chronic fatigue is likely the symptom of CCSVI.

In addition, it is estimated that the lifetime risk of depression in MS patients is as high as $50 \%$, compared to $10 \%$ in the general population. The prevalence of major depression is also significantly higher in MS compared to any other chronic disease [12-14]. Prevalence of anxiety is found to be around $50 \%$ in MS patients $[12,15]$.

In the present study, we investigated the correlation between anomalous venous outflow and the seriousness of fatigue and anxiety as well as depression in MS patients and age- and sex-matched healthy controls.

\section{Patients and Methods}

\section{Patients}

Twenty MS patients of the outpatient department of the St. Antonius MS centre, Nieuwegein, The Netherlands, were included in this study. All had been diagnosed with MS using the revised McDonald's criteria [16]. Twelve patients were diagnosed with RRMS, 4 with PPMS and 4 with secondary progressive MS (SPMS). 
The EDSS ranged from 1.5 to 6.5, with a median of 3.0. Twelve of the patients were currently on immunomodulating medication (interferon beta- 1 a or -1 b, glatiramer acetate, natalizumab or intravenous immunoglobulins).

Twenty age- and sex-matched healthy controls underwent the same investigations as the MS patients. The study was approved by the Ethics Committee of our institution (Verenigde Commissies Mensgebonden Onderzoek), and informed consent was obtained from all participating subjects.

\section{Colour Doppler Sonography}

All patients and controls were investigated using extra- and transcranial Colour Doppler ultrasonography (3-9 MHz linear array transducer and 1-4 MHz sector array transducer, respectively, on Philips iU22 ultrasound system; Philips Healthcare, Eindhoven, The Netherlands). All 40 subjects were evaluated by one of the two experienced and trained sonographers in the presence of a clinical neurophysiologist (S.C.T. who is certified in neurosonology by the European Society of Neurosonology and Cerebral Hemodynamics, and attended the course named 'Echo Doppler in the Diagnosis of CCSVI', Ferrara, Italy). The sonographer and clinical neurophysiologist were blinded for patients and controls. A third person helped the patient or volunteer on the examination couch and in the right position, so the sonographer and clinical neurophysiologist were 'blinded' for the MS patients and healthy volunteers. In each patient and control, the internal jugular vein (IJV) and vertebral vein (VV) were evaluated on both sides and in both the supine and sitting position. The basal vein of Rosenthal (BVR) was evaluated in the supine position alone. Five parameters related to the impaired cerebral venous outflow were tested [1]:

(1) No detectable flow in the IJV and/or the VV in both the supine and sitting position.

(2) Reflux in the IJV or VV in both the supine and sitting position. Reflux was defined as a reversal of the direction of the venous blood flow during normal unforced inhalation and exhalation, and during a short period of apnoea following a normal exhalation. Reflux was defined as a flow directed toward the brain for a duration of $>0.88 \mathrm{~s}$.

(3) Evidence of IJV stenosis using the B mode.

(4) A higher cross-sectional area of the IJV in the sitting position as compared to the supine position, indicating loss of postural regulation of the main cerebrospinal venous outflow pathways.

(5) Flow other than unidirectional in the BVR. This was evaluated through the temporal bone window, if present.

Fatigue Severity Scale and Hospital Anxiety Depression Subscale

To determine the level of fatigue, anxiety and depression all patients and volunteers filled out the Fatigue Severity Scale (FSS) and the Hospital Anxiety Depression Subscale (HADS).

The FSS [17] consists of nine items each scored from 1 to 7 (i.e. from 'strongly disagree' to 'strongly agree'). It covers physical, social, or cognitive effects of fatigue. The FSS score is the total score of the nine individual items. The minimal score is 9 and the maximal possible score is 63. A higher score indicates a greater fatigue severity. A total score $\geq 36$ is indicative of significant fatigue.

The HADS [18] consists of seven questions relating to anxiety and seven questions relating to depression. Each question can be scored from 0 to 3 (i.e. from 'not at all' to 'very often indeed'). Each subscale has a minimal score of 0 and a maximum score of 21 . A higher score indicates a greater depression or anxiety severity. A score $\geq 16$ is valued as severe depression or anxiety.

\section{Statistical Analysis}

Statistical analysis was performed using the Mann-Whitney U test with SPSS 18.0. Results were determined as statistically significant at $\mathrm{p}<0.05$.

\section{Results}

The median age in the patient group was 41.5 years compared to 44.5 years in the healthy controls (not significant). Both groups consisted of 8 males and 12 females. Baseline characteristics of the study population are presented in table 1. 
Table 1. Baseline characteristics of patients with MS and healthy controls

Table 2. Cerebral venous outflow anomalies in MS patients and healthy controls

\begin{tabular}{|c|c|c|c|}
\hline Variable & $\begin{array}{l}\text { MS } \\
\text { patients } \\
(n=20)\end{array}$ & $\begin{array}{l}\text { Controls } \\
(\mathrm{n}=20)\end{array}$ & $\mathrm{p}$ value \\
\hline (1) No flow detectable in IJV or VV & $4(20)$ & $1(5)$ & \\
\hline (2) Reflux in IJV or VV & $1(5)$ & 0 & \\
\hline (3) IJV stenosis & $4(20)$ & $1(5)$ & \\
\hline $\begin{array}{l}\text { (4) Higher cross-sectional area of } \\
\text { IJV in the sitting as compared } \\
\text { to the supine position }\end{array}$ & 0 & $2(10)$ & \\
\hline (5) No unidirectional flow in BVR & 0 & 0 & \\
\hline $\begin{array}{l}\text { Total number of patients with at } \\
\text { least one abnormal parameter }\end{array}$ & $8(40)$ & $3(15)$ & 0.183 \\
\hline $\begin{array}{l}\text { Total number of patients with at } \\
\text { least two abnormal parameters }\end{array}$ & $1(5)$ & $2(10)$ & 0.799 \\
\hline
\end{tabular}

In total, nine abnormal CCSVI parameters were found in 8 MS patients (table 2). In addition, five abnormal CCSVI parameters were found in 3 healthy controls (not significant). The anomalies that were most frequently found were the absence of flow in the IJV or VV in the sitting or supine position and a stenosis detected in the IJV. One RRMS patient had an arteriovenous fistula between the left IJV and the common carotid artery. One SPMS patient was found to have both a reflux in the left IJV and a stenosis of the right IJV. In one healthy control, stenosis was seen in both the left and right IJV.

The level of fatigue was significantly higher in the MS group compared to the healthy volunteers ( 46.0 vs. $18.5, \mathrm{p}<0.01$ ). The severity of anxiety and depression was also significantly greater in the MS population: on the HADS, the MS group had a median anxiety score of 5.5 compared to 4.0 in the control group $(p<0.05)$ and a depression score of 4.5 compared to 1.0 in the control group ( $\mathrm{p}<0.05$ ). Only 1 RRMS patient had a score $\geq 16$ on the HADS for anxiety as well as depression.

Of the MS patients with abnormal parameters, the median FSS score was 47.5, whereas the median FSS score in MS patients without abnormal parameters was $41.5(p=0.706)$. The median score on the HADS anxiety subscale was 6.5 for MS patients with venous outflow anomalies and 5 for MS patients without abnormal parameters $(p=0.205)$. On the HADS 
Hagens et al:: Cerebrospinal Venous Outflow in Multiple Sclerosis Patients versus Fatigue and/or Depression

Table 3. Median fatigue, anxiety and depression scores in subjects with or without cerebral venous outflow anomalies

\begin{tabular}{|c|c|c|c|c|c|c|c|c|c|c|c|c|}
\hline \multirow[t]{2}{*}{ Variable } & \multicolumn{4}{|l|}{ MS } & \multicolumn{4}{|l|}{ Controls } & \multicolumn{4}{|c|}{ Total (MS patients and controls) } \\
\hline & $\begin{array}{l}\text { total } \\
(\mathrm{n}=20)\end{array}$ & $\begin{array}{l}\text { normal } \\
(\mathrm{n}=12)\end{array}$ & $\begin{array}{l}\text { anomalies } \\
(\mathrm{n}=8)\end{array}$ & p value & $\begin{array}{l}\text { total } \\
(\mathrm{n}=20)\end{array}$ & $\begin{array}{l}\text { normal } \\
(\mathrm{n}=17)\end{array}$ & $\begin{array}{l}\text { anomalies } \\
(\mathrm{n}=3)\end{array}$ & p value & $\begin{array}{l}\text { total } \\
(\mathrm{n}=40)\end{array}$ & $\begin{array}{l}\text { normal } \\
(n=29)\end{array}$ & $\begin{array}{l}\text { anomalies } \\
(\mathrm{n}=11)\end{array}$ & $\mathrm{p}$ value \\
\hline \multicolumn{13}{|l|}{ Median } \\
\hline FSS & 46 & 41.5 & 47.5 & 0.706 & 18.5 & 18 & 21 & 0.765 & 27.5 & 25 & 43 & 0.090 \\
\hline \multicolumn{13}{|l|}{ Median } \\
\hline $\begin{array}{l}\text { HADS } \\
\text { anxiety }\end{array}$ & 5.5 & 5 & 6.5 & 0.205 & 4 & 4 & 2 & 0.216 & 4 & 4 & 6 & 0.455 \\
\hline \multicolumn{13}{|l|}{ Median } \\
\hline HADS & & & & & & & & & & & & \\
\hline depression & 4.5 & 4 & 5 & 0.795 & 1 & 1 & 1 & 0.921 & 2 & 2 & 5 & 0.402 \\
\hline
\end{tabular}

Normal $=$ Patients and/or controls without cerebral venous outflow anomalies; anomalies = patients and/or controls with cerebral venous outflow anomalies.

depression subscale, the median score for MS patients without impaired cerebrospinal venous outflow was 5 , compared to 4 in MS patients with normal venous outflow $(\mathrm{p}=0.795$; table 3).

In the healthy controls, the FSS and HADS scores also did not differ significantly between subjects with or without cerebrospinal venous outflow anomalies (table 3).

Furthermore, we formed two subgroups according to the presence or absence of venous outflow anomalies irrespective of the presence of MS or not. There were no significant differences in the FSS of the two subgroups and the HADS between the groups with or without abnormal ultrasound parameters.

\section{Discussion}

The results of the present study show no significantly impaired cerebrospinal venous outflow in patients with MS versus sex- and age-matched controls. In addition, impaired venous outflow was even found in 3 healthy controls. The definition of CCSVI is commonly defined as two or more abnormal venous outflow parameters $[2,19]$. Only 1 patient in the MS group met this criterion compared to 2 of the controls. This invalidates the hypothesised correlation between patients with MS and impaired cerebrospinal venous outflow. These results are in line with the outcomes of multiple studies worldwide in which no relation between impaired cerebrospinal venous outflow and MS was found [20-27]. It is also in agreement with the outcome of the large cohorts investigated so far, the observational study of the prevalence of CCSVI in MS and in other neurodegenerative diseases (CoSMo study) [28].

In the present study, we investigated the relationship between impaired cerebrospinal venous outflow and fatigue and depression. As expected, the median FSS and HADS scores were significantly higher in the MS group compared to the healthy control group. This corresponds to the high levels of fatigue, anxiety and depression found in MS patients in various publications [7-10, 12-15]. However, we did not find any significant correlation between fatigue as measured by the FSS and impaired cerebrospinal venous outflow in MS patients. This finding is in strong contrast with the findings of Malagoni et al. [11]: in their study, they 
found a significant reduction in chronic fatigue after balloon dilatation of stenosing lesions affecting the main extracranial veins. They suggest that patients with impaired cerebrospinal venous outflow experience a higher rate of perceived fatigue than patients without impaired cerebrospinal venous outflow, a finding we cannot confirm in our study.

In addition, no significant correlation was found between anxiety and/or depression, as measured by the HADS, and impaired cerebrospinal venous outflow in MS patients. Moreover, when combining the subjects with or without cerebrospinal venous outflow anomalies from both the MS patients and the healthy controls, there was no significant difference in the scores on the FSS or HADS between the two combined groups. Altogether, we cannot confirm the suggested relation between impaired cerebrospinal venous outflow in MS patients and fatigue, anxiety or depression.

We are aware of the relevant limitations of this study, mainly the small sample size. Both the MS group and the control group are rather small, but our outcomes are in line with previously published studies in which no significant correlations between impaired cerebrospinal venous outflow and MS or between impaired cerebrospinal venous outflow and the (overall) quality of life in MS patients have been reported. Even when combining the MS patients and healthy controls with cerebrospinal venous outflow anomalies, we cannot confirm a significant relation between impaired cerebrospinal venous outflow and quality of life as measured by the HADS and FSS. As there seems to be no significant difference between impaired cerebrospinal venous outflow and fatigue, depression or anxiety before any treatment, it is questionable if endovascular treatment of such impaired cerebrospinal venous outflow would have a significant effect on fatigue, depression or anxiety in MS patients. Our outcomes do not support the hypothesis that endovascular treatment of venous outflow anomalies would improve the quality of life in MS patients. Such significant effect was indeed not found in a number of other studies [4-6].

The strong relation between venous outflow anomalies and MS found in other studies might (at least in part) be explained by the varying methodological quality of a number of these studies. A key factor is blinding of sonographers and evaluators, and in case of treatment of the impaired venous outflow, also blinding of the patients. Not all studies attempted blinding and some had a low success of blinding in more severe MS patients. We tried to solve this problem by asking a third person to position the patient. As the maximum EDSS in our patient population was as low as 6.5, all patients were able to walk for a short distance. After the patient was positioned by the third person, the sonographer and the clinical neurophysiologist were not able to differentiate between a MS patient or a healthy control subject. To avoid any suggestion of MS by a possible speech or language disorder, the patients and controls were not allowed to speak at any time in the presence of the sonographer or the clinical neurophysiologist.

Besides the problem of blinding, extra- and transcranial Colour Doppler sonography is subject to a high degree of interobserver variation. A lower level of experience and training of the sonographer could result in higher levels of false-negative or false-positive outcomes. Using multiple different sonographers in one study could increase this interobserver variation. This might in part explain the variation in the prevalence of impaired cerebrospinal venous outflow in MS patients in different studies. Our examinations were performed by only two well-trained and highly experienced sonographers and only one clinical neurophysiologist to limit interrater and intrarater variations as much as possible.

In conclusion, we found no significantly impaired cerebrospinal venous outflow in patients with MS versus sex- and age-matched controls. Furthermore, we cannot confirm the hypothesised relation between impaired cerebrospinal venous outflow in MS patients and fatigue, anxiety or depression suggesting again that there is no indication for endovascular treatment for impaired cerebrovenous outflow in MS patients. 
Hagens et al:: Cerebrospinal Venous Outflow in Multiple Sclerosis Patients versus Fatigue and/or Depression

\section{Acknowledgements}

We thank L. Teunissen, M. Wols van der Wel-van Egdom and P. Sibbes-Dekker for their help in blinding and performing the ultrasound examinations.

This study was supported by grant 10-699MS of the MS Research Foundation, Voorschoten, The Netherlands.

\section{Disclosure Statement}

The authors have no conflicts of interest to disclose.

\section{References}

1 Zamboni P, Galeotti R, Menegatti E, et al: Chronic cerebrospinal venous insufficiency in patients with multiple sclerosis. J Neurol Neurosurg Psychiatry 2009;80:392-399.

-2 Zamboni P, Consorti G, Galeotti R, et al: Venous collateral circulation of the extracranial cerebrospinal outflow routes. Curr Neurovasc Res 2009;6:204-212.

-3 Laupacis A, Lillie E, Dueck A, et al: Association between chronic cerebrospinal venous insufficiency and multiple sclerosis: a meta-analysis. CMAJ 2011;183:E1203-E1212.

4 Ghezzi A, Annovazzi P, Cocco E, et al: Endovascular treatment of CCSVI in patients with multiple sclerosis: clinical outcome of 462 cases. Neurol Sci 2013;34:1633-1637.

5 Hubbard D, Ponec D, Gooding J, Saxon R, Sauder H, Haacke M: Clinical improvement after extracranial venoplasty in multiple sclerosis. J Vasc Interv Radiol 2012;23:1302-1308.

-6 Zagaglia S, Balestrini S, Perticaroli E, et al: Percutaneous transluminal angioplasty for chronic cerebrospinal venous insufficiency in multiple sclerosis: dichotomy between subjective and objective outcome scores. Neurol Sci 2013;34:2205-2210.

7 Bakshi R: Fatigue associated with multiple sclerosis: diagnosis, impact and management. Mult Scler 2003;9: 219-227.

8 Bergamaschi R, Romani A, Versino M, Poli R, Cosi V: Clinical aspects of fatigue in multiple sclerosis. Funct Neurol 1997;12:247-251.

-9 Fisk JD, Pontefract A, Ritvo PG, Archibald CJ, Murray TJ: The impact of fatigue on patients with multiple sclerosis. Can J Neurol Sci 1994;21:9-14.

10 Freal JE, Kraft GH, Coryell JK: Symptomatic fatigue in multiple sclerosis. Arch Phys Med Rehabil 1984;65: 135-138.

11 Malagoni AM, Galeotti R, Menegatti E, et al: Is chronic fatigue the symptom of venous insufficiency associated with multiple sclerosis? A longitudinal pilot study. Int Angiol 2010;29:176-182.

12 Jones KH, Ford DV, Jones PA, et al: A large-scale study of anxiety and depression in people with Multiple Sclerosis: a survey via the web portal of the UK MS Register. PLoS One 2012;7:e41910.

$\checkmark 13$ Goldman Consensus Group: The Goldman Consensus statement on depression in multiple sclerosis. Mult Scler 2005;11:328-337.

14 Patten SB, Beck CA, Williams JV, Barbui C, Metz LM: Major depression in multiple sclerosis: a population-based perspective. Neurology 2003;61:1524-1527.

15 Janssens AC, van Doorn PA, de Boer JB, et al: Anxiety and depression influence the relation between disability status and quality of life in multiple sclerosis. Mult Scler 2003;9:397-403.

16 Polman $\mathrm{CH}$, Reingold SC, Banwell B, et al: Diagnostic criteria for multiple sclerosis: 2010 revisions to the McDonald criteria. Ann Neurol 2011;69:292-302.

$\checkmark 17$ Krupp LB, LaRocca NG, Muir-Nash J, Steinberg AD: The fatigue severity scale. Application to patients with multiple sclerosis and systemic lupus erythematosus. Arch Neurol 1989;46:1121-1123.

18 Zigmond AS, Snaith RP: The hospital anxiety and depression scale. Acta Psychiatr Scand 1983;67:361-370.

19 Zivadinov R, Marr K, Cutter G, et al: Prevalence, sensitivity, and specificity of chronic cerebrospinal venous insufficiency in MS. Neurology 2011;77:138-144.

-20 Baracchini C, Perini P, Causin F, Calabrese M, Rinaldi F, Gallo P: Progressive multiple sclerosis is not associated with chronic cerebrospinal venous insufficiency. Neurology 2011;77:844-850.

21 Barreto AD, Brod SA, Bui TT, et al: Chronic cerebrospinal venous insufficiency: case-control neurosonography results. Ann Neurol 2013;73:721-728.

22 Blinkenberg M, Akeson P, Sillesen H, et al: Chronic cerebrospinal venous insufficiency and venous stenoses in multiple sclerosis. Acta Neurol Scand 2012;126:421-427.

23 Doepp F, Wurfel JT, Pfueller CF, et al: Venous drainage in multiple sclerosis: a combined MRI and ultrasound study. Neurology 2011;77:1745-1751. 
24 Leone MA, Raymkulova 0, Naldi P, et al: Chronic cerebrospinal venous insufficiency is not associated with multiple sclerosis and its severity: a blind-verified study. PLoS One 2013;8:e56031.

25 Mayer CA, Pfeilschifter W, Lorenz MW, et al: The perfect crime? CCSVI not leaving a trace in MS. J Neurol Neurosurg Psychiatry 2011;82:436-440.

26 Tsivgoulis G, Mantatzis M, Bogiatzi C, et al: Extracranial venous hemodynamics in multiple sclerosis: a casecontrol study. Neurology 2011;77:1241-1245.

27 Wattjes MP, van Oosten BW, de Graaf WL, et al: No association of abnormal cranial venous drainage with multiple sclerosis: a magnetic resonance venography and flow-quantification study. J Neurol Neurosurg Psychiatry 2011;82:429-435.

28 Comi G, Battaglia MA, Bertolotto A, et al: Observational case-control study of the prevalence of chronic cerebrospinal venous insufficiency in multiple sclerosis: results from the CoSMo study. Mult Scler 2013;19:15081517. 